\title{
A New Method for the Economic Laws of Extinction Using the Fox-Wright-type Function
}

\author{
Rabha W. Ibrahim ${ }^{1 *}$, Samir B. Hadid ${ }^{2}$ \\ ${ }^{1 *}$ University Malaya, 50603, Malaysia \\ ${ }^{2}$ Ajman University of Science and Technology, UAE
}

Received 13 April 2018; accepted 12 September 2018; available online 19 September 2018

DOI: https://10.30880/jst.2018.10.03.002

\begin{abstract}
In this note, we deal with the possibility of optimal economic extinction. We employ the FoxWright-type function to characterize the probability of transference from optimal selection to the economic laws of extinction. For the extinction, we shall utilize the fractional Poisson process.
\end{abstract}

Keyword: Fractional calculus; fractional differential operator; fractional differential equation; extinction.

\section{Introduction}

Economics defines the selection of goods (services) as a matching performance between the cost and benefit of producing the next item. The next item of goods (services) is that the economists discuss to describe a marginal optimal for capable decision making. In good markets, the cost of producing the next item (marginal costs) are normally increasing over the related range of manufacture, and the marginal benefits to the fixed are equal to the fixed price established per unit of production. While in the demand direction, traditional economics equally frames the choice of how much to expend as a matching performance between marginal cost and marginal benefits from expending. Marginal benefits are much more challenging to measure and are regularly based on the economist's idea of expending utility.

As an expending increase, the quantity of utility is assumed to be diminishing to capture a notion of increasing fulfillment at a decreasing rate. How much to expand is evaluated by the equating marginal utility with the market price. This traditional economic observation of optimal manufacture, optimal expands, and market exchange has been expanded to contain goods without a welldefined market price. This holds when the right cost, the optimal manufacture and expanding of market and non-market goods presents the most extensive system of independents and choice possible. There are different methods to evaluate the utility function. Recently, the author suggested a generalization utility function by using fractional calculus and fractional entropy [1]-[3].

The economic laws of extinction are optimal behavior under a procedure of the individual offering at a point in time to maximize profit or utility. In other words, if the marginal cost (price) is less than the marginal benefit, then the type will be determined as an optimal extinction. Extinction can occur with sufficient client demand and a high positive time preference under a market device of distribution. In economic languages, the occasion costs can become too high when compared to insatiable client demand and increasing incomes.

In this effort, we deal with the possibility of optimal economic extinction. We employ the Fox-Wright-type function to characterize the probability of transference from an optimal selection of extinction rule in economic systems. For the extinction, we shall utilize the fractional Poisson process.

\section{Processing}

In this section, we need three fractional power series to complete our algorithm.

\subsection{Fractional Poisson process}

Based on the definition of the RiemannLiouville fractional calculus [4] 
${ }_{a} D_{t}^{\wp} v(t)=\frac{d^{n+1}}{d t^{n+1}} \int_{a}^{t} \frac{(t-\tau)^{n-\wp}}{\Gamma(n-\wp)} v(\tau) d \tau, \quad n \leq \wp<n+1$

Laskin [5] introduced a generalization of the Poisson distribution. The probability of the extinction is provided in term of fractional Poisson process as follows:

$P_{\wp}(k, \xi)=\frac{\left(\mu \xi^{\wp}\right)^{k}}{k !} \sum_{n=0}^{\infty} \frac{(n+k) !}{n !} \frac{\left(-\mu \xi^{\wp}\right)^{n}}{\Gamma(\wp(n+k)+1)}, \quad \wp \in(0,1]$

where $\mu$ is the single Poisson parameter that is utilized to set the Poisson distribution, $\xi$ is the Poisson random variable and $\Gamma$ is the Euler's Gamma function. For $k=0$, the above equation becomes

$P_{\wp}(0, \xi)=\sum_{n=0}^{\infty} \frac{\left(-\mu \xi^{\wp}\right)^{n}}{\Gamma(\wp n+1)}=E_{\wp}\left(-\mu \xi^{\wp}\right)$

where $E_{\rho}\left(-\mu \xi^{\rho}\right)$ is the Mittag-Leffler function of order $\rho$ [4]. Consequently, the fractional extinction rule is given by

$$
Q(\xi):=P_{\rho}(0, \xi)=E_{\rho}\left(-\mu \xi^{\zeta \rho}\right)
$$

\subsection{Mittag-Leffler function}

The Mittag-Leffler function $E_{a, b}$ is a special complex function which depends on two complex parameters $a$ and $b$. It can be represented by the following series:

$E_{a, b}(\varsigma)=\sum_{n=0}^{\infty} \frac{\varsigma^{n}}{\Gamma(a n+b)}$

The Mittag-Leffler function has an asymptotic power, rule attitude and the fractional calculus presents the historical influence. This explanation proposes the fractional forming as a good tool to define without practical hypotheses, complex power, rule performance and systems with long range associations. Moreover, the Mittag-Leffler function indicates slower distribution that the exponential function, this brings to an infinite mean free path distribution (the mean free path is the average distance moved by a shifting the economy and the microeconomy). Beginning from this method, and considering that FoxWright, general functions are promptly related to irregular diffusion processes, we propose an applicable solution for the issue of the economic laws of extinction.

\subsection{Fox-Wright function}

The Fox-Wright function is a generalization of the hypergeometric function as follows

${ }_{p} \Psi_{q}\left[\begin{array}{llll}\left(\alpha_{1}, \Lambda_{1}\right) & \left(\alpha_{2}, \Lambda_{2}\right) & \ldots & \left(\alpha_{p}, \Lambda_{p}\right) \\ \left(\beta_{1}, \Upsilon_{1}\right) & \left(\beta_{2}, \Upsilon_{2}\right) & \ldots & \left(\beta_{q}, \Upsilon_{q}\right)\end{array} ; \xi\right]=\sum_{n=0}^{\infty} \frac{\Gamma\left(\alpha_{1}+\Lambda_{1} n\right) \cdots \Gamma\left(\alpha_{p}+\Lambda_{p} n\right)}{\Gamma\left(\beta_{1}+\Upsilon_{1} n\right) \cdots \Gamma\left(\beta_{q}+\Upsilon_{q} n\right)} \frac{\xi^{n}}{n !}$

For the shifting economic system, the probability can be presented by a special type of the Fox-Wright function as follows:

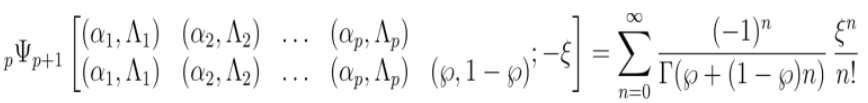

By using the term $\Gamma(\rho)$ as a normalized factor, we consider the probability of nonnegative random variables $\xi \in \Xi$ as follows:

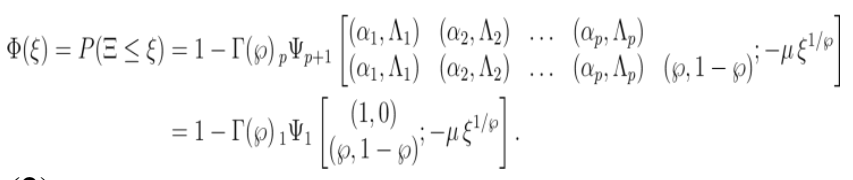

Eq. (2) shows the attitude of the transmission probability (ATP) of economy in the range $\rho \in$ $[0,0.8)$. It can be seen that the function $\Phi(\xi)$ is sub-exponential function in the rang $\xi \in(0,2]$ and super-exponential in $\xi \geq 3$. This ATP will increase the client utility, where some economic models are simulated on maximizing client utility. Basically, this is measured by the flow of per-capita consumption. Consequently, one of the most dominant inferences from economics is that this flow must be sufficiently differentiated. This model can be represented as ecosystem processes. Species extinction and biodiversity loss are probably under a market pattern of optimal and marginal transformation.

\subsection{Preliminaries}

To provide some properties of our algorithm, we request the Ramanujan Master Theorem.

Lemma 2.1: For a function $\varphi(y)$ that in the neighborhood of the origin satisfies the form

$\phi(y)=\sum_{n=0}^{\infty} \varphi(n) \frac{(-1)^{n} y^{n}}{n !}$,

such that $\phi(0) 6=0$, then 


$$
\int_{0}^{\infty} d y y^{\nu-1} \phi(y)=\Gamma(\nu) \varphi(-\nu), \quad \nu \in \mathbb{R}
$$

\section{Results}

Economic and social systems have a complex relation. This relation achieves non linearity in both time and deterministic. Traditional economics have faced toward the equilibrium and steady. The behavior of the economic extinction process obeys to (2) has range $\rho \in$ $[0.8,1)$ (see Fig 1). Let us assume a numerical method, where $N(\xi), \xi \geq 0$, denotes the number of obstacles in economies that have existed in the space $\Xi$ satisfying (2).

Proposition 3.2: (Free path distribution) FPD The explicit formula of Eq. (2) is

$\overline{\Phi(\xi)}:=-\frac{\mu \xi^{1 / \wp-1}}{\wp} \Phi(\xi)$

Proof 1: Following [6] the definition of FPD is given as follow:

$d P(\xi)=\left|\frac{d P_{\wp}(k, \xi)}{d \xi}\right| d \xi$.

Applying the Poisson process formula, we have

$$
\begin{aligned}
\frac{d P_{\wp}(k, \xi)}{d \xi} d \xi & =\Gamma(\wp) \frac{d}{d \xi}\left(\sum_{n=0}^{\infty} \frac{(-1)^{n} \mu^{n} \xi^{n / \wp}}{n ! \Gamma(-\wp n+n+\wp)}\right) d \xi \\
& =\frac{\Gamma(\wp)}{\wp}\left(\sum_{n=0}^{\infty} \frac{(-1)^{n} \mu^{n} \xi^{n / \wp-1}}{n ! \Gamma(-\wp n+n+\wp)}\right) d \xi \\
& =\frac{\mu \xi^{1 / \wp-1} \Gamma(\wp)}{\wp}\left(\sum_{n=0}^{\infty} \frac{(-1)^{n+1} \mu^{n} \xi^{n / \wp}}{n ! \Gamma((1-\wp) n+1)}\right) d \xi \\
& =-\frac{\mu \xi^{1 / \wp-1}}{\wp} \Phi(\xi) d \xi .
\end{aligned}
$$

Proposition 3.3: (Mean Free path distribution) MFPD The mean free path distribution of Eq.(2) can be calculated by the form

$\gamma_{\wp}=\frac{1}{\mu^{\wp}} \frac{\Gamma(\wp) \Gamma(\wp+1)}{\Gamma\left(\wp^{2}\right)}$.

By the definition of the MFPD, we have Proof 2:

$$
\gamma_{\wp}:=\frac{\int_{0}^{\infty} \xi d P_{\wp}(k, \xi)}{\int_{0}^{\infty} d P_{\wp}(k, \xi)}=\frac{\int_{0}^{\infty} \frac{\mu \xi^{1 / \wp}}{\wp} \Phi(\xi)}{\int_{0}^{\infty} \frac{\mu \xi^{1 / \wp-1}}{\wp} \Phi(\xi)} .
$$

Assume that $z=\mu \xi^{1 / \rho}$ then we have

$$
\int_{0}^{\infty} \frac{\mu \xi^{1 / \wp}}{\wp} \Phi(\xi) d \xi=\frac{1}{\mu^{\wp}} \int_{0}^{\infty} z^{\wp} \Phi(z) d z .
$$

In view of Lemma 2.1, we arrive at

$\frac{1}{\mu^{\wp}} \int_{0}^{\infty} z^{\wp} \Phi(z) d z=\frac{1}{\mu^{\wp}} \frac{\Gamma(\wp) \Gamma(\wp+1)}{\Gamma\left(\wp^{2}\right)}$

and for the integral

$$
\int_{0}^{\infty} \frac{\mu \xi^{1 / \wp-1}}{\wp} \Phi(\xi)=1
$$

Hence, we have the desired equation.

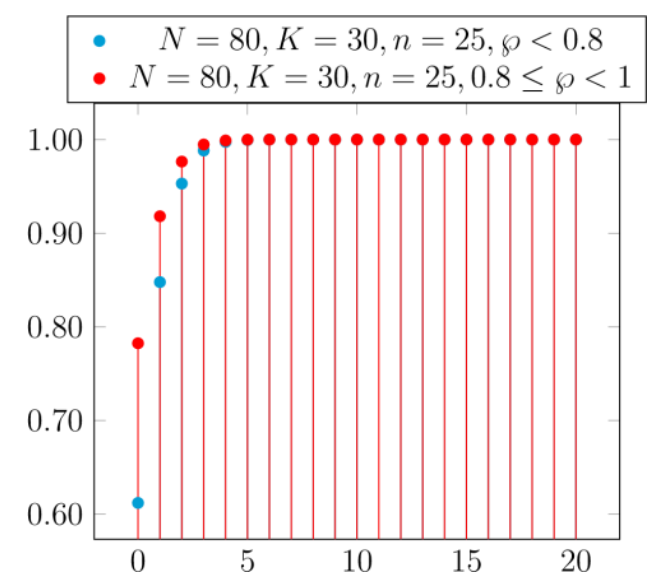

Figure 1: The Fox-Wright function of order $0<$ $\rho<1$ in Eq. (2). The probability approaches slowly to one, when $\rho<0.8$; rather than $0.8<$ $\rho<1$ for example at $\xi=0$ the probability is 0.8 for $\rho=0.8$ and is equal to 0.6 for $\rho=0.5$.

\section{Conclusion}

Utilizing a generalized Fox-Wright function in (ATP) of economy, we reach to the relation as

$$
\gamma_{\wp} \rightarrow \frac{1}{\mu^{\wp}}, \quad \wp \rightarrow 1
$$

This process provides extinction process in economic modeling in terms of the following equation 


$$
\gamma_{\wp}=\frac{1}{\mu^{\wp}} \frac{\Gamma(\wp) \Gamma(\wp+1)}{\Gamma\left(\wp^{2}\right)}
$$

The deviation can be realized by the difference:

$$
\Delta(\xi)=Q(\xi)-\Phi(\xi)
$$

This leads to consider sub-exponential and super-exponential deviation from (1) and (2). Consequently, this can relate to the diffusion in economy (technological change). Eq. (3) can be represented to the rate of acceptance of technology, which is calculated by factors such as ease of use and usefulness. Moreover, (3) can be viewed as a comparison between two processes, achieving the sub-exponential and super-exponential properties. Fig.2 shows that the deviation $\Delta(\xi)$ decreases when $\xi \in[1,5)$ and converges to 0 , whenever $\xi \geq 5$. Our method was Lemma 2.1, with the coefficient values.

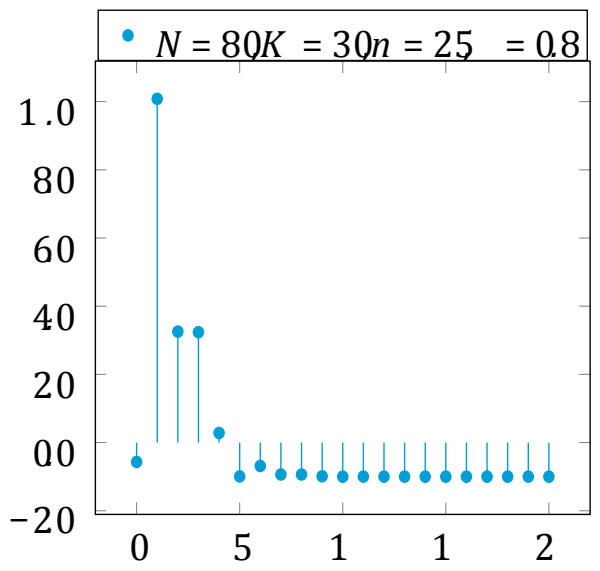

Figure 2: The deviation $\Delta(\xi), 0<\rho<1$ in Eq.(3). $\Delta(\xi) \rightarrow 0$ for $\xi \geq 5, \rho=0.8$

$$
\varphi(n)=\frac{1}{\Gamma((1-\wp) n+1)}, \quad \varphi(0) \neq 0
$$

Furthermore, Eq. (3) can recognize as a steady-state economy. This equation basically indicates the national or local economy. In this case, the economy can approximate to the steady- state after a period of growth or after a period of downsizing or regrowth. Finally, Eq. (3) may be indicating the convergence of our method.

\section{Acknowledgements}

The author has no conflict of interest with other parties.

\section{References}

[1] Ibrahim, RW. et al. (2016) Perturbation of fractional multi-agent systems in cloud entropy computing. Entropy, Vol.18. No.1pp 31

[2] Ibrahim, RW. \& Gani, A. (2016) Hybrid cloud entropy systems based on Wiener process, Kybernetes, Vol. 45. No. 7 pp. 1072-1083.

[3] Ibrahim, RW. \& Salih, YK. (2017) On a fractional multi-agent cloud computing system based on the criteria of the existence of fractional differential equation, Mathematical Sciences, Vol 2017, No. 1, pp 1-7

[4] Podlubny, I. (1999) Fractional differential equations. New York: Academic Press.

[5] Laskin, NF. (2003) Fractional Poisson process, Commun Nonlinear Sci Numer Simulation, Vol 8, No 1, pp 201-13.

[6] Gorska, K. et al. (2012) The Ramanujan master theorem and its implications for special functions, App. Math. Comput. Vol 218, No. 1, pp11466-11471. 